\title{
SMOOTH BANACH SPACES ${ }^{1}$
}

\author{
BY KONDAGUNTA SUNDARESAN ${ }^{2}$
}

Communicated by V. Klee, January 3, 1966

1. Introduction. The purpose of this note is the study of twice differentiability of the norm in a real Banach space. We establish the various properties of the second derivative and obtain a polar characterization of twice differentiability of the norm. As a consequence of the various results a characterization of Hilbert spaces among Banach spaces which may be equipped with an equivalent twice differentiable norm is obtained.

2. Notations and definitions. Throughout this note $E$ denotes a real Banach space with a Fréchet differentiable norm so that the spherical image map $G$ on the unit sphere $S$ of $E$ into $S^{*}$, the unit sphere of $E^{*}$ (the dual of $E$ ) is a function. For complete details and references about the first order differentiability of the norm in $E$ in relation to the function $G$ we refer to Cudia [1]. If $x \in S$ then $E_{x}$ denotes the closed subspace $G(x)^{-1}(0)$.

Definition. Let $(E,\|\cdot\|)$ be a Banach space. Then the norm is said to be twice differentiable at $x \neq 0$ if there exists a symmetric bilinear functional $T_{x}$ on $E \times E$ such that

$$
\|x+h\|=\|x\|+G(x) h+T_{x}(h, h)+\theta_{x}(h)
$$

where $\theta_{x}(h) /\|h\|^{2} \rightarrow 0$ as $\|h\| \rightarrow 0$ and $G(x)$ is the Gateux derivative of the norm at $x$. If the norm is twice differentiable at all members in $S$ then the Banach space $E$ is said to be twice Fréchet differentiable. The functional $T_{x}$ may be identified as a bounded operator on $E$ into $E^{*}$ by the formula $\sigma\left(T_{x}\right)(y) z=T_{x}(y, z)$.

With the above notations we obtain the following theorems.

THEOREM 1. If the norm of the Banach space $E$ is twice differentiable at $x$ then

(i) the norm is twice differentiable at all members $\lambda x, \lambda \neq 0$ and $T_{\lambda x}=T_{x} /|\lambda|$.

(ii) $T_{x}(y, y) \geqq 0$ for all $y \in E$ and

(iii) the range of the operator $\sigma\left(T_{x}\right) \subseteq\{x\}^{\perp}$.

1 This note is a summary of the first part of a dissertation submitted in partial fulfillment of the requirements for the Ph.D. degree at the University of Washington, Seattle.

${ }^{2}$ Author supported in part by contract NSF GP-378. 
As a consequence of the above theorem we obtain the following polar characterization for the norm in $E$ to be twice differentiable at a member $x \in S$.

THEOREM 2. The norm in $E$ is twice Fréchet differentiable at a member $x \in S$ if and only if the mapping $\phi$ defined on $E_{x}$ into $\{x\}^{\perp}$ defined by setting $\phi(h)=\pi_{x} \circ G(x+h)$ where $\pi_{x}$ is the projection on $E^{*}$ into the closed subspace $Q x^{-1}(0), Q x$ being the canonical image of $x$ in $E^{* *}$, is Frechet differentiable.

As a consequence of the above theorems we obtain the following isomorphism theorems.

TheOREM 3. If the norm in $E$ is twice differentiable at an element $x \neq 0$ and if the restriction of the operator $\sigma\left(T_{x}\right)$ to $E_{x}$ is an isomorphism then $E$ is isomorphic to a strictly convex Banach space. Further if $\inf _{y \in E_{x} \cap s} T_{x}(y, y)>0$ then $E$ is isomorphic to a Hilbert space.

THEOREM 4. If the Banach space $E$ and its dual $E^{*}$ are twice differentiable then $E$ is isomorphic to a Hilbert space.

The relationship between the twice differentiability of the norm and various notions of metric curvature [2] and the existence of free tangents [3] to a class of arcs on the unit sphere and the proofs of the above theorems will be appearing elsewhere.

\section{REFERENCES}

1. D. F. Cudia, The geometry of Banach spaces, smoothness, Trans. Amer. Math. Soc. 110 (1964), 283-314.

2. L. M. Blumenthal, Theory and application of distance geometry, Oxford at the Clarendon Press, 1953.

3. G. Ewald and L. M. Kelly, Tangents in real Banach spaces, J. Reine Angew. Math. 203 (1960) 160-173.

UNIVERSITY OF WASHINGTON AND

Carnegie Institute of Technology 